UCRL-JC-126866

PREPRINT

\title{
Nonequilibrium Detonation of Composite Explosives
}

\author{
Albert L. Nichols III \\ This paper was prepared for submittal to the \\ 1997 Topical Conference on Shock Compression of Condensed Matter \\ of the American Physical Society \\ Amherst, MA \\ July 27 - August 1, 1997
}

July 1997

This is a preprint of a paper intended for publication in a journal or proceedings. Since changes may be made before publication, this preprint is made available with the understanding that it will not be cited or reproduced without the permission of the author. 


\section{DISCLAIMER}

This document was prepared as an account of work sponsored by an agency of the United States Government. Neither the United States Government nor the University of California nor any of their employees, makes any warranty, express or implied, or assumes any legal liability or responsibility for the accuracy, completeness, or usefulness of any information, apparatus, product, or process

disclosed, or represents that its use would not infringe privately owned rights. Reference herein to any specific commercial product, process, or service by trade name, trademark, manufacturer, or otherwise, does not necessarily constitute or imply its endorsement, recommendation, or favoring by the United States Government or the University of California. The views and opinions of authors expressed herein do not necessarily state or reflect those of the United States Government or the University of California, and shall not be used for advertising or product endorsement purposes. 


\title{
NONEQUILIBRIUM DETONATION OF COMPOSITE EXPLOSIVES
}

\author{
Albert L. Nichols III \\ Lawrence Livermore National Laboratory \\ L-282, PO Box 808, Livermore, CA 94550
}

The effect of nonequilibrium diffusional flow on detonation velocities in composite explosives is examined. Detonation conditions are derived for complete equilibrium, temperature and pressure equilibrium, and two forms of pressure equilibrium. Partial equilibria are associated with systems which have not had sufficient time for transport to smooth out the gradients between spatially separate regions. The nonequilibrium detonation conditions are implemented in the CHEQ equation of state code. We show that the detonation velocity decreases as the non-chemical degrees of freedom of the explosive are allowed to equilibrate. It is only when the chemical degrees of freedom are allowed to equilibrate that the detonation velocity increases.

\section{INTRODUCTION}

The detonation properties of uniform materials has a long history in the literature. Less is understood about the properties of mixtures of explosives. In this paper I will examine some of the issues involved with considering the detonation properties of mixed systems within a pseudo Chapmann-Jouget approach.

It is clear from experiments by McGuire et.al. (1) that a mixed explosive need not go to complete chemical equilibration, even after completing the entire detonation process. By examining several mechanisms for non-ideal detonation, it is hoped that we might understand the actual extent of equilibration possible within the detonation front.

There are several mechanisms which can lead to non-ideal behavior in a composite explosive which are not present in an homogeneous explosive. In general, the composite explosives will tend to have different compositions and would have different detonation temperatures if they were by themselves. They would also have different particle and shock velocities. We four states that the heterogeneous system can progress through:

1. Pressure equilibrium

2. Pressure and particle velocity equilibrium

3. Thermal but not chemical equilibrium

4. Complete equilibrium

\section{THEORY}




\section{Thermal Equilibration}

We will not go into the derivation of the standard Chapman-Jouget Detonation theory here. The standard CJ conditions for the detonation of a thermally equilibrated composite explosive are: Particle Velocity (Conservation of Mass):

$$
u=D\left(1-\frac{\left\langle v_{i}\right\rangle}{\left\langle v_{i}^{0}\right\rangle}\right)
$$

Detonation Velocity (Conservation of Momentum):

$$
D^{2}=\frac{\left\langle v_{i}^{0}\right\rangle^{2}\left(P-P^{0}\right)}{\left\langle v_{i}^{0}-v_{i}\right\rangle},
$$

Hugoniot relation (Conservation of Energy):

$$
\left\langle e_{i}-e_{i}^{0}\right\rangle=\frac{1}{2}\left(P+P^{0}\right)\left\langle v_{i}^{0}-v_{i}\right\rangle .
$$

Here $u$ is the particle velocity, $D$ is the detonation velocity, $P$ is the pressure, $v$ is the volume per unit mass, and $e$ is the energy per unit mass. The CJ state is the state which minimizes the entropy of the entire system. Here the \langle\rangle imply a mass fraction $\left(x_{i}\right)$ weighted sum over the $i$ components.

For complete chemical equilibrium, the product components are homogenized and we can neglect the initial substructure. For thermal equilibrium, we assume that

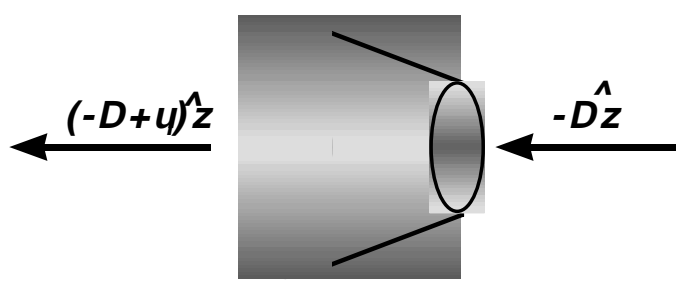

FIGURE 1. An example of a volume element of one of the explosives in the detonation front. The detonation wave is progressing from left to right. the composition of each explosive does not change and we can use the preceding equations without further modification.

\section{Pressure Equilibration Only}

In a composite explosive, the only things which are ensured to be uniform throughout the system are the detonation velocity and pressure. To analyze this situation, it is necessary to use the integral form of the conservation equations. It is assumed that the volume relative to other components of the explosive need not be fixed as it detonates, i.e. one explosive can expand while the other contracts.

If we assume that the volume of integration follows the flow of the material through the detonation, as shown in Fig 1, then the contributions to the conservation equations from the sides will be zero for both the conservation of mass and energy. There will be a contribution due to the conservation of momentum which depends on the rate pressure changes as the system goes through the detonation front. For this work, we will neglect that ill-defined term.

Given these assumptions we find:

Particle velocity of each component:

$$
u_{i}=D\left(1-\frac{\rho^{0} x_{i}^{0}}{\rho x_{i}}\right) \text {. }
$$

Detonation velocity:

$$
D^{2}=\frac{\left(P-P^{0}\right)}{\rho^{0}\left[1-\left\langle\left(\frac{x_{i}^{0} \rho^{0}}{x_{i} \rho}\right)\right\rangle^{0}\right]}
$$




$$
\rho x_{i}=\frac{\rho^{0} x_{i}^{0}}{2}\left\{\frac{\rho_{i} D^{2}}{P}+\frac{P^{0} \rho_{i}}{P \rho_{i}^{0}}\left[\left(\frac{\rho_{i} D^{2}}{P}+\frac{P^{0} \rho_{i}}{P \rho_{i}^{0}}\right)^{2}-4 \frac{\rho_{i} D^{2}}{P}\right)^{\frac{1}{2}}\right\}
$$

Effective Hugoniot Relation for each component:

$$
\left(e_{i}-e_{i}^{0}\right)=\frac{1}{2}\left(v^{0}-v \frac{x_{i}^{0}}{x_{i}}\right)\left[P\left(2 \frac{v_{i} x_{i}}{v x_{i}^{0}}-R_{i}\right)+P^{0} R_{i}\right]
$$

where:

$$
R_{i}=\left(1-\frac{v x_{i}^{0}}{v^{0} x_{i}}\right) /\left(1-\left\langle\frac{v x_{i}^{0}}{v^{0} x_{i}}\right\rangle^{0}\right)
$$

and where \langle\rangle$^{\circ}$ is the mass fraction average over the initial mass fraction and $\rho$ is the density. Note that the average in Eq. (5) will result in an increase in the detonation velocity for a given initial composition.

Particle velocity equilibration occurs as the relative velocities between the two explosives are dissipated by viscous effects. A uniform particle velocity requires that the mass fractions not change across the detonation front. This allows us to simplify the effective Hugoniot condition to:

$$
\left(e_{i}-e_{i}^{0}\right)=\frac{1}{2}\left(v^{0}-v\right)\left[P\left(2 \frac{v_{i}}{v}-1\right)+P^{0}\right]
$$

\section{IMPLEMENTATION AND RESULTS}

All calculations were conducted with the chemical equilibrium-equation of state code CHEQ. $(2,3,4,5)$ CHEQ calculates the equilibrium composition at a given temperature and pressure by minimizing the Gibb's free energy. CHEQ incorporates an effective one-component fluid variational-perturbation treatment of high-temperature, high-pressure, multi-component fluids. This model has been applied to the study of the equation of state of detonation products.(3) CHEQ has equation of state models for three carbon phases (diamond, graphite, and liquid) developed by van Thiel and Ree.(4)

The non-thermally equilibrated states described here require a more complicated method of solution. To calculate the pressure only equilibrium the Hugoniot of the completely equilibrated state at a specified pressure is calculated. This provides an initial values for the function

$$
\lambda=\frac{P}{\rho^{0} D^{2}}
$$

and the component densities. The mixed detonation velocity and mole fractions are then calculated self-consistently to determine $\lambda$. $\lambda$ is used to determine the detonation velocity which is used in the subsequent solutions of Eq (6). This process is continued until the values of $\lambda$ between successive iterations converge. This gives the state on the pressure equilibrium only Hugoniot curve at the specified pressure. A series of these calculations are performed until the detonation velocity is minimized.

For the pressure and particle velocity equilibrium, CHEQ uses the same initial state as used in the pressure only equilibrium case. It uses that state to get an initial estimate of the density at the detonation condition. CHEQ then solves Eq (8) for each species. A new estimate for the density is then determined and then $\mathrm{Eq}(8)$ is solved again until the densities converge. A succession of these states is calculated until the detonation velocity is minimized.

For purposes of these calculations, two explosives with significantly different chemical composition were chosen. The 


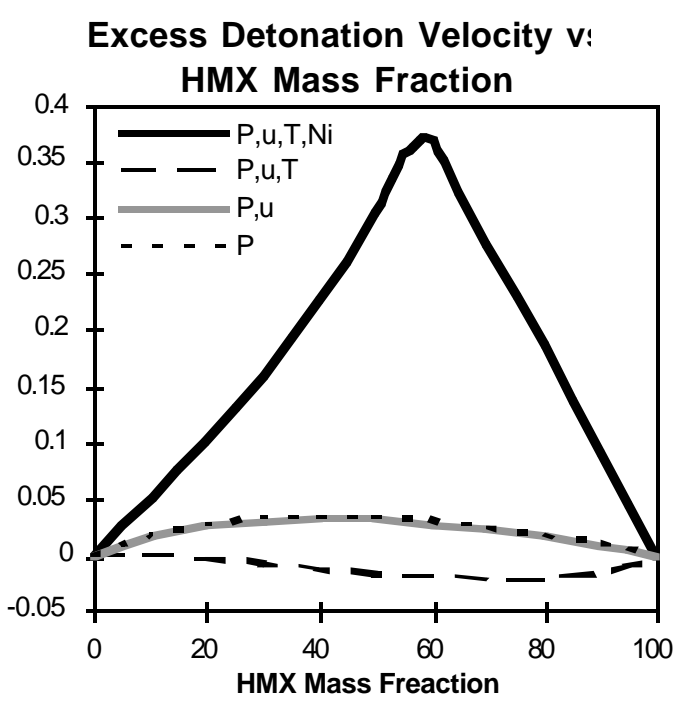

FIGURE 2. The difference in the detonation velocity in an HMX/ADN explosive between a straight baseline and various non-equilibrium assumptions, as a function of the weight percent of HMX.

first is HMX. The density, atomic composition, and heat of formation are $1.89 \mathrm{gm} / \mathrm{cm}^{3}, \mathrm{C}_{4} \mathrm{H}_{8} \mathrm{~N}_{8} \mathrm{O}_{8}$, and 75.02 $\mathrm{kJ} / \mathrm{mol}$, respectively. The main products from this explosive are nitrogen, water, and either carbon monoxide or carbon dioxide and some form of solid carbon. In general, if it is burned, the products have more of the monoxide, while if it detonates it produces more of the dioxide. The second explosive that was chosen is ADN. The density, atomic composition, and heat of formation are $1.803 \mathrm{gm} / \mathrm{cm}^{3}, \mathrm{H}_{4} \mathrm{~N}_{4} \mathrm{O}_{4}$, and $-149.787 \mathrm{~kJ} / \mathrm{mol}$, respectively. In contrast to $H M X$ which is under oxidized, $A D N$ is over oxidized. This implies that the detonation products have a significant amount of molecular oxygen. It also has absolutely no carbon.

The CHEQ calculations described here used the fluid species $\mathrm{H}_{2}, \mathrm{O}_{2}, \mathrm{H}_{2} \mathrm{O}, \mathrm{CH}_{4}, \mathrm{CO}$, $\mathrm{CO}_{2}, \mathrm{~N}, \mathrm{~N}_{2}, \mathrm{NH}_{3}, \mathrm{NO}, \mathrm{NO}_{2}, \mathrm{~N}_{2} \mathrm{O}$, and $\mathrm{CHOOH}$, and three phases of carbon: diamond, graphite, and liquid. Two fluid phases were used for the non-carbon fluid species. That is, each fluid species was allowed to have a concentration in two fluid phases. By including two fluid phases, the fluid system can exhibit a super-critical phase separate into nitrogen rich and water rich phases, the existence of which has been postulated by Ree (3).

For those systems where it is assumed that the composition has not equilibrated, a complete set of species are assigned to each explosive component. The composition is allowed to equilibrate within each component's set of species, but the composition is not allowed to migrate between the two component's sets of species.

In Figure 2 we show the effect of the non-equilibrium behavior by comparing the difference of the detonation velocities as a function of composition from a straight baseline connecting the two extremes of detonation velocity.

\section{CONCLUSION}

In this paper we showed three nonequilibrium cases of detonation and compared them to the standard equilibrium detonation case. The three non-equilibrium states correspond to assumptions regarding the rates of equilibration of kinetic, thermal, and material inhomogeneities, with kinetic equilibration being the fastest process, and material equilibration being the slowest. We note that the detonation velocity need not be a monatonic function of the extent of equilibrium.

In order to verify the conclusions of this work, one must be able to vary the extent of equilibrium in a composite explosive. One way of doing this would be 
in changing the particle size of the monomolecular explosive components in the composite explosive. As the particles become smaller the mixture of the two components become more intimate, implying that the range of heterogeneity is smaller. This smaller range would allow the viscous and diffusive effects to dissipate more of the non-uniformity in the detonation products. Therefore, we expect that the detonation velocity of a composite explosive will be a nonmonatonic function of the particle size.

The pressure only equilibrium case is only possible in a composite explosive where the explosives have been laid out in parallel strands and when the detonation is traveling in a direction parallel to the strands. If the detonation were traveling in any other direction, the components with a higher particle velocity will plow directly into those components with slower velocities, thus rapidly transferring the momentum from one component to the other. When the detonation is traveling parallel to the strands, though, the detonation can set up a standing three dimensional structure where the only momentum which is passed is in the plane of the detonation wave.

There is much evidence that would indicate that even monomolecular explosives fail to attain full equilibrium. We have shown that there are at least three mechanisms in composite explosives beyond those found in the monomolecular explosives which can result in a nonequilibrium detonation. We have also proposed how these mechanisms might be examined with simple detonation tests.
The author would like to thank Craig Tarver, Edward Lee, Mathias van Thiel, and Francis Ree for helpful discussions and encouragement.

This work performed under the auspices of the U.S. Department of Energy by the Lawrence Livermore National Laboratory under contract number W-7405-Eng48 .

\section{BIBLIOGRAPHY}

1 McGuire, R.R., Ornellas, D.L., Akst, I.B., Propellants and Explosives 4, 23-29 (1979).

2 Ree, F. H., J. Chem. Phys., 78, 409, (1983).

3 Ree, F. H., J. Chem. Phys., 81, 1251, (1984).

4 van Thiel, M., and Ree., F. H., High Pressure Research, 10, 607 (1992).

5 Nichols, A.L. III, and Ree, F.H., Lawrence Livermore National Laboratory, UCRL-MA-106754, (1990)

\section{ACKNOWLEDGMENTS}




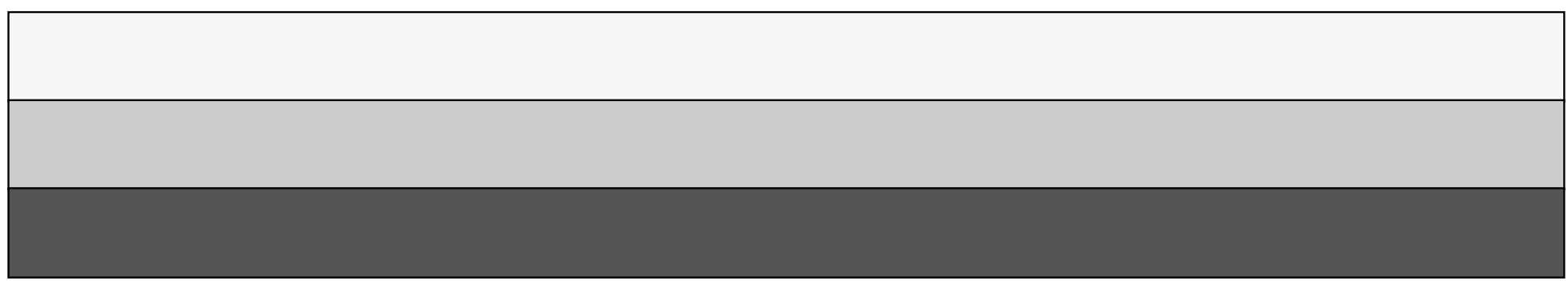

